\title{
Impact of CFO on Low Latency-Enabled UAV using "Better than Nyquist" Pulse Shaping in GFDM
}

\author{
Navuday Sharma ${ }^{1}$, Atul Kumar ${ }^{2}$, Maurizio Magarini ${ }^{2}$, Stefano Bregni ${ }^{2}$, Dushantha Nalin K. Jayakody ${ }^{3,4}$
}

\begin{abstract}
Low altitude aerial base stations onboard unmanned aerial vehicles (UAVs) have recently gained a lot of attention to provide cellular coverage to mobile ground users. In this paper we evaluate the effect of carrier frequency offset (CFO) on the symbol error rate in the downlink of such systems, where a long-term evolution (LTE) compatible time-frequency grid based on generalized frequency division multiplexing (GFDM) is considered. The choice of GFDM as multi-carrier modulation scheme is motivated by its widely proposed application in fifth generation cellular systems and its backward compatibility with LTE. Simulation results are shown for "Better than the Nyquist (BTN)" pulse shaping filters in three different environments: Suburban, Urban and Urban High Rise. The use of BTN pulse shaping filters allows for higher robustness to CFO as compared to base line pulse shaping filer know as root-raised cosine filters for different environments conditions. Simulation results are presented considering realistic air-to-ground propagation conditions generated from the commercial Wireless InSite raytracing radio propagation software.
\end{abstract}

Index Terms-Aerial base station; air-to-ground communications; pulse shaping filters; carrier frequency offset; generalized frequency-division multiplexing; symbol error rate.

\section{INTRODUCTION}

IMT-2020 cellular systems, commonly known as 5G, offer high data rate, ultra-reliable and low latency communications [1]. For fulfilling high data rate demands, unmanned aerial vehicles (UAVs), working as aerial base stations (ABSs) have recently received a lot of interest [2], [3]. Essentially, ABSs are highly useful for servicing flash crowds such as festivals, carnivals, musical concerts, sport events etc., where large group of people demand for high data rates [4]. This also conducts to compact and small cell architectures, leading to the concept of ultra-dense cloud drone networks [5].

synchronization impairments on transmission performance. In [6], the bit error rate (BER) of an IEEE 802.11a compatible orthogonal frequency division multiplexing (OFDM) system that includes Doppler shift and inter-carrier interference (ICI) is analyzed. In [7] the authors study a UAV-enabled downlink orthogonal frequency-division multiple access (OFDMA) network by taking into account delay-specified minimum-rateratio constraints of the users. The minimum user throughput is maximized by jointly optimizing the UAV trajectory and the

${ }^{1}$ Tallin University of Technology, Estonia, ${ }^{2}$ Politecnico di Milano, Italy, ${ }^{3}$ School of Computer Science and Robotics, National Research Tomsk Polytechnic University, Russia,and ${ }^{4}$ School of Postgraduate Studies, Sri Lanka Technological Campus Sri Lanka. Email: navuday.sharma@taltech.ee, \{atul.kumar,maurizio.magarini,stefano.bregni\}@ polimi.it,

nalin.jayakody@ieee.org. This work was done when the first author, Navuday Sharma, was with Tomsk Polytechnic University, Russia and Politecnico di Milano, Italy communication resource allocation. In [8] the authors present the waveform design of an OFDM system with customizable modulation parameters. The implementation of a self-designed hybrid software defined radio (SDR) platform is discussed for a UAV acting as a relay between an unmanned ground vehicle and a ground control station. In [9] an OFDM waveform and spectrum management scheme is proposed. To efficiently operate multiple UAVs by different operators, [10] proposes a centralized radio resource management assuming OFDM and a time-frequency grid of the LTE system. The work in [11], focuses on channel estimation and ICI mitigation for moderate-to-high Doppler.

However, to the best of our knowledge, there is no existing literature about implementation of generalized waveforms to support high data rate requirements of the users in the ABS downlink. Thus, in this article we study generalized frequency division multiplexing (GFDM) with low latency communication parameters in a LTE time-frequency grid with compatible transmission time interval (TTI) based frame structure [12]. The transmission of multiple sub-symbols on the same subcarrier in GFDM allows to reduce the number of sub-carriers as compared to OFDM based on the same TTI.

As in [13], we resort to Monte Carlo simulations to measure the symbol error rate (SER) at different altitudes of the ABS. The air-to-ground (A2G) channel parameters obtained in [14] with ray-tracing are used. "Better than Nyquist" (BTN) pulse shaping filters are considered in our simulations, as proposed in [15], due to the improved error rate performance, less outof-band emission as compared to Nyquist pulses, and less sensitivity to carrier frequency offset (CFO). As is well known, CFO causes attenuation in the desired signal and introduces ICI. It results mainly from a frequency mismatch between transmitter and receiver local oscillators and Doppler shifts.

The main advantages of LAPs are improved SER with better line-of-sight (LoS) probability and lower path loss (PL). Also, we do not consider the case of moving ABSs since the users are in flash crowds, where large number of people are present in a small area [4]. Such a scenario would be well served by a static UAV, with the possibility of hovering close to the users while maintaining a safe distance. Simulations for GFDM are performed in three different scenarios, namely suburban, urban and urban high rise buildings, by using A2G channel parameters obtained from ray-tracing at $2.4 \mathrm{GHz}$. We implement the system model in sub- $6 \mathrm{GHz}$ band as preferred in 3GPP [16], where various field trials have been conducted by companies. Not that the unlicensed spectrum are considered in our simulations since a dedicated frequency band has not been standardized yet in 3GPP. It is worth observing that 
WiFi also operates in the same frequency band. However, for vehicular communication, WiFi-based physical layer is challenging to provide reliable communication [17]. Also from [17], it is clear that GFDM performs better than the OFDM with WiFi parameters due to better temporal and spectral efficiency and higher throughput. This confirms that GFDM has better performance in the $2.4 \mathrm{GHz}$ unlicensed band.

The paper is organized as follows. Section II defines the GFDM system and the A2G channel model. Pulse shaping filters are given in Sec. III. The ray tracing simulation setup is given in Sec. IV. Section V provides the SER simulation results with $\mathrm{CFO}$ and $\mathrm{BTN}$ pulse shaping at different ABSs altitudes. Finally, conclusions are drawn in Sec. VI.

\section{System ModeL}

In this work, the GFDM system is implemented as given in [18]. The vector of $N=K M$ transmitted symbols is

$$
\mathbf{d}=\left[\left(\boldsymbol{d}_{0}\right)^{T},\left(\mathbf{d}_{1}\right)^{T}, \ldots \ldots,\left(\mathbf{d}_{K-1}\right)^{T}\right]^{T},
$$

with

$$
\mathbf{d}_{k}=\left[d_{k, 0}, d_{k, 1}, \ldots \ldots, d_{k, M-1}\right]^{T},
$$

where $d_{k, m}$ is the $m$ th sub-symbol transmitted on $k$ th subcarrier and $(\cdot)^{T}$ is the transpose operator. The time-duration of each data block $\mathbf{d}_{k}$ is $M T_{s}$ and the sub-carrier spacing is equal to $1 / T_{s}$, where $T_{s}$ is sub-symbol duration associated with the data at the input.

The transmitted signal is modeled as

$$
x[n]=\sum_{k=0}^{K-1} \sum_{m=0}^{M-1} d_{k, m} g_{k, m}[n], n=0,1, \ldots \ldots K M-1 .
$$

with

$$
g_{k, m}[n]=g[(n-m K) \bmod N] e^{\frac{-\mathrm{j} 2 \pi \mathrm{kn}}{K}},
$$

where $g[n]$ is the prototype filter, $\bmod N$ is the modulo $N$ operation, which makes $g_{k, m}[n]$ a circularly shifted version of $g_{k, 0}[n]$, and the exponential function performs the frequency shifting operation.

The vector representation of (1) can be written by collecting the $N$ samples of $g_{k, m}[n]$ in the matrix form as

$$
\mathbf{x}=\mathbf{A} \mathbf{d},
$$

where $\mathbf{x}=[x[0], x[1], \cdots, x[N-1]]^{T}$, and $\mathbf{A}=\left[\boldsymbol{g}_{0,0} \cdots\right.$ $\left.\boldsymbol{g}_{K-1,0} \boldsymbol{g}_{0,1} \boldsymbol{g}_{K-1,1} \boldsymbol{g}_{0, M-1} \ldots \boldsymbol{g}_{K-1, M-1}\right]$. Before transmission, a cyclic prefix (CP) of length $N_{C P}$ is added to form the vector $\widehat{\boldsymbol{x}}=\left[\mathbf{x}\left(N-N_{C P}: N-1\right)^{T}, \mathbf{x}^{T}\right]^{T}$. The length of $\mathrm{CP}$ is taken to be the same as the number of channel taps in our simulations.

The transmission over a multipath $\mathrm{A} 2 \mathrm{G}$ probabilistic channel with path loss and large scale fading effects can be modeles as

$$
\mathrm{PL}_{\mathrm{LoS}}(\mathbf{d})[\mathrm{dB}]=20 \log _{10}\left(\frac{4 \pi \mathbf{d}_{\mathbf{0}}}{\lambda}\right)+10 \eta_{\mathrm{LoS}} \log _{10}(\mathbf{d})+X_{\sigma, \mathrm{LoS}},
$$

$\mathrm{PL}_{\mathrm{NLoS}}(\mathbf{d})[\mathrm{dB}]=20 \log _{10}\left(\frac{4 \pi \mathbf{d}_{\mathbf{0}}}{\lambda}\right)+10 \eta_{\mathrm{NLoS}} \log _{10}(\mathbf{d})+X_{\sigma, \mathrm{NLoS}}$,

where LoS and NLoS represent line of sight and non-LoS, respectively, with PL denoting the path loss, $\eta$ the path loss exponent, $\mathbf{d}$ is the distance between ABS and ground receiver with $\mathbf{d}_{\mathbf{0}}$ be the reference distance, here assumed as $\mathbf{d}_{\mathbf{0}}=1$. $X_{\sigma}$ is the log-normal random variable with standard deviation $\sigma$ which includes the large scale fading effects. The average path loss model can be obtained as [19]

$$
\operatorname{PL}(d)[\mathrm{dB}]=\mathbb{P}_{\mathrm{LoS}} \cdot \mathrm{PL}_{\mathrm{LoS}}(d)+\left(1-\mathbb{P}_{\mathrm{LoS}}\right) \cdot \mathrm{PL}_{\mathrm{NLoS}}(d),
$$

where

$$
\mathbb{P}_{\text {LoS }}=\frac{1}{1+p \exp (-q[\phi-p])}
$$

is the probability to have a LoS link, being $p$ and $q$ the parameters of the LoS curve, which are related to the environment planning parameters, $\alpha, \beta$ and $\gamma$ :

- $\alpha=$ Ratio of land area covered by the buildings to the total area (dimensionless).

- $\beta=$ Number of buildings per unit area (building/ sq km).

- $\gamma=$ Variable determining the building height distribution.

The PL values for the considered environments at different ABS heights can be found in [14]. However, this model does not include the multipath, which is usually modeled as Ricean for the A2G channel [20]. The discrete time impulse response $h[n]$ can be obtained from stochastic approach through empirical data, geometric analysis, and simulations [20]. Here we describe the multipath channel as

$$
h[n]=\sum_{k=0}^{L-1} h_{k} \delta[n-k],
$$

where $h_{k}$ is the Ricean faded $k$ th complex coefficient obtained from ray tracing data as $\mathrm{PG}[\mathrm{dB}]=\mathrm{PL}(d)[\mathrm{dB}]-\mathrm{PL}_{\text {tot }}(d)[\mathrm{dB}]$ and $L$ denotes the number of channel taps as given in Table I. PG represents the path gain of each multipath component or channel tap and $\mathrm{PL}_{\text {tot }}(d)[\mathrm{dB}]$ is the total path loss including both large and small scale fading effects. At the receiver side of the GFDM, the length of CP must be higher than the maximum delay spread of the multipath channel, i.e, $N_{C P} \geq L$. Under these assumptions, the received signal vector after removal of the $\mathrm{CP}$ is

$$
\mathbf{y}=e^{\frac{j 2 \pi \epsilon n}{K}} \mathbf{H} \mathbf{x}+\mathbf{w},
$$

where $\epsilon=\Delta f M T_{S}$ is the CFO $\Delta f$ normalized to the subcarrier spacing $1 / M T_{S}$, being $T_{S}$ the symbol interval and $\mathbf{H}$ a circulant matrix of size $N \times N$ based on the $N \times 1$ vector h whose first $L$ elements coincide with the impulse response of the channel and the remaining $N-L$ elements are zero. The vector $\mathbf{w}$ is the noise vector of size $N \times 1$ in which each entry is an i.i.d. zero mean complex Gaussian random variable with variance $\sigma_{w}^{2}$. Here, matched filtering (MF) receiver is considered. The equalized signal in time domain is

$$
\hat{\mathbf{d}}=\mathbf{B}_{M F} \mathbf{y}_{e q}=\mathbf{B}_{M F} \mathbf{A d}+\mathbf{B}_{M F} \mathbf{H}^{-1} \mathbf{w},
$$

where $\boldsymbol{B}_{M F}=\left(\boldsymbol{A}^{H} \boldsymbol{A}\right)^{-1} \boldsymbol{A}^{H}$ and $\mathbf{y}_{e q}$ is the equalized signal. The pulse shaping filter $g[n]$ in the GFDM system affects the 
spectral efficiency and the performance. A standard approach for choosing the pulse shaping filter is to sample a continuoustime impulse response $g(t)$ windowed as

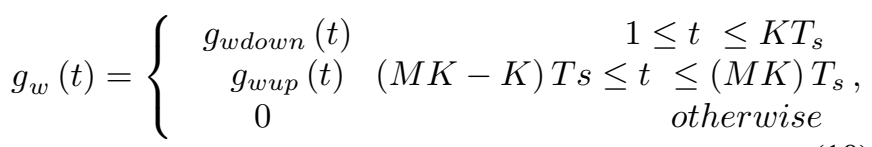

where $g_{\text {wup }}(t)=g_{\text {pulse }}(t), g_{\text {wdown }}(t)=1-g_{\text {pulse }}(t)$ and $T_{s}$ symbol interval, being $g_{\text {pulse }}(t)$ one of the different types of pulse shaping filters reported below.

\section{Pulse Shaping Filter}

In this section, we define the pulse shaping filters used for performance evaluation with CFO in the GFDM system model. Moreover, we consider the time-domain expression that results by interchanging the independent frequency variable with the time variable for all pulse shaping filter given below.

\section{A. Root raised cosine ( $R R C)$}

The time domain expression of the root raised cosine (RRC) for a given roll-off factor $\alpha$ is given as

$$
g_{R R C}(t)=\sqrt{g_{R C}(t)} .
$$

where

$g_{R C}(t)=\left\{\begin{array}{l}1, \quad|t| \leq \frac{(1-\alpha) T_{s}}{2} \\ \frac{1}{2}\left[1+\cos \left(\pi P_{R C}(t)\right)\right], \frac{(1-\alpha) T_{s}}{2}<|t| \leq \frac{(1+\alpha) T_{s}}{2} \\ 0, \quad \text { otherwise, }\end{array}\right.$

being $P_{R C}(t)$ is the inner argument of the cosine give in [15].

\section{B. Flipped-hyperbolic secant (Fsech)}

This improved Nyquist pulse shaping filter is proposed in [21]. By following [21], we consider first the flippedhyperbolic secant (Fsech) pulse shaping filters define as

$$
g(t)=\left\{\begin{array}{c}
1, \quad|t| \leq \frac{(1-\alpha) T_{s}}{2} \\
1-\operatorname{sech}\left(\rho P_{1 F \operatorname{sech}}(t)\right), \frac{(1-\alpha) T_{s}}{2}<|t| \leq \frac{T_{s}}{2} \\
\operatorname{sech}\left(\rho P_{2 F \operatorname{sech}}(t)\right), \frac{\frac{T_{s}}{2}<|t| \leq \frac{(1+\alpha) T_{s}}{2}}{2}<,
\end{array}\right.
$$

where sech is the hyperbolic secant function, $\rho=$ $\ln (\sqrt{3}+2) / \alpha \times \frac{T_{s}}{2}, \quad P_{1 F \text { sech }}(t)$ and $P_{2 F \text { sech }}(t)$ is the inner arguments of the hyperbolic secant function given in [15].
TABLE I

Power Delay PROFILE AT ABS ALTITUde of 500M

\begin{tabular}{|l|l|l|l|l|l|}
\hline \multicolumn{2}{|l|}{ Suburban } & Urban & \multicolumn{2}{l|}{ Urban High Rise } \\
\hline $\begin{array}{l}\text { Delay } \\
\text { (in ns) }\end{array}$ & $\begin{array}{l}\text { Power } \\
\text { (in dB) }\end{array}$ & $\begin{array}{l}\text { Delay } \\
\text { (in ns) }\end{array}$ & $\begin{array}{l}\text { Power } \\
\text { (in dB) }\end{array}$ & $\begin{array}{l}\text { Delay } \\
\text { (in ns) }\end{array}$ & $\begin{array}{l}\text { Power } \\
\text { (in dB) }\end{array}$ \\
\hline 1703 & -50.5 & 1715 & 3.7 & 1759 & 113 \\
\hline 1935 & 9.9 & 1737 & -65.4 & 1942 & 99.1 \\
\hline 2123 & 5.8 & 1752 & -80.6 & 2293 & 85.2 \\
\hline 2324 & 15.6 & 1784 & -66.9 & 2307 & 78.9 \\
\hline 2352 & -12.4 & 2257 & -26.6 & 2335 & 115.4 \\
\hline 2504 & 27.8 & 2294 & -4.1 & 2513 & 76.7 \\
\hline 2627 & -47.6 & 2321 & -35.2 & 2676 & 47.3 \\
\hline 2689 & 3.27 & 2524 & -59.8 & 2723 & 42.4 \\
\hline 2892 & -28 & 2695 & -13.3 & 2906 & 98.1 \\
\hline 2911 & 9.3 & 2691 & 12.8 & 3005 & 130 \\
\hline
\end{tabular}

\section{Flipped-inverse hyperbolic secant (Farcsech)}

Another improved Nyquist pulse shaping filter proposed in [21] is the flipped-inverse hyperbolic secant (Farcsech) define as

$g(t)=\left\{\begin{array}{c}1, \quad|t| \leq \frac{(1-\alpha) T_{s}}{2} \\ \operatorname{arcsech}\left(\frac{1}{\rho} P_{1 F \operatorname{arcsech}}(t)\right), \quad \frac{(1-\alpha) T_{s}}{2}<|t| \leq \frac{T_{s}}{2} \\ 1-\operatorname{arcsech}\left(\frac{1}{\rho} P_{2 F \operatorname{sech}}(t)\right), \frac{T_{s}}{2}<|t| \leq \frac{(1+\alpha) T_{s}}{2} \\ 0, \\ \frac{(1+\alpha) T_{s}}{2}<|t|,\end{array}\right.$

where arcsech is the inverse hyperbolic secant function, $P_{1 \text { Farcsech }}(t)$ and $P_{2 \text { Fsech }}(t)$ is the inner arguments of the inverse hyperbolic secant are give in [15].

\section{RAY TRACing Simulation Setup}

The simulation results of this paper were performed by a commercial radio propagation software, Wireless InSite, to obtain various A2G channel parameters [14] at different ABS altitudes, which were used in SER simulations at corresponding $\mathrm{ABS}$ altitudes. The ray tracing simulations were carried out in three different generalized environments- suburban, urban and urban high rise, due to which the channel characteristics can be related to realistic scenarios. These environments were created in a computer-aided design (CAD) software, 3DS Max according to ITU-R parameters [19] $\alpha, \beta$ and $\gamma$, as given in Sec. II. The simulation area was $1000 \times 1000 \mathrm{~m}^{2}$, with 32,500 receivers uniformly distributed on the ground and the ABS placed at the center. The density of the buildings depends on the type of environment, with suburban having the least density and urban high rise having the most, while the heights of the buildings were Rayleigh distributed [22]. The altitude of ABS was varied from 100 to $2000 \mathrm{~m}$. Three simulations were carried out at different ABS height intervals of $100 \mathrm{~m}$ at $2.4 \mathrm{GHz}$ carrier frequency and $20 \mathrm{MHz}$ signal bandwidth, with a fixed transmission power of ABS at $18 \mathrm{dBm}$. The results were averaged at each height and two snapshots at different ABS locations were taken to improve their accuracy. The unlicensed spectrum was preferred for this work since no dedicated spectrum has been allocated for the ABS network till now. Also, isotropic antenna was used at ABS to remove the 
TABLE II

PARAMETERS FOR GFDM SIMULATION WITH LTE GRID

\begin{tabular}{|c|c|}
\hline Parameter & Normal mode \\
\hline Subframe duration & $1 \mathrm{~ms}$ or 30.720 samples \\
\hline GFDM symbol duration & $66.67 \mu \mathrm{s}$ or 2048 samples \\
\hline Sub-symbol duration & $4.17 \mu \mathrm{s}$ or $128 \mathrm{samples}$ \\
\hline Sub-carrier spacing & $240 \mathrm{KHz}$ \\
\hline Sub-carrier bandwidth & $240 \mathrm{KHz}$ \\
\hline Sampling freq. (clock) & $30.72 \mathrm{MHz}$ \\
\hline Sub-carrier spacing factor $N$ & 128 \\
\hline Sub-symbol spacing $K$ & 128 \\
\hline active sub-carriers $N_{\text {on }}$ & 75 \\
\hline Sub-symbols per GFDM symbol $M$ & 15 \\
\hline GFDM symbols per subframe & 15 \\
\hline CP length & $4.17 \mu$ s or 128 samples \\
\hline
\end{tabular}

effects due to antenna directivity. Two snapshots of simulations were captured in different parts of the environments for improving the precision of the results. The results of these simulations were averaged at corresponding heights to increase the accuracy of the results. Wireless InSite provides accurate results as from comparison with measurements [23].

\section{Simulation Results}

The SER analysis was carried out using three BTN pulse shaping filters - Flipped-hyperbolic secant (Fsech), Flippedinverse hyperbolic secant (Farcsech) and Reverse- Farcsech (R-Farcsech) in the GFDM system with variation in CFO and ABS altitude in different environments. We have used Low latency PHY simulation parameters as shown in Table II. In this section, we present our results in three categories to obtain an optimal ABS altitude and pulse shaping filter.

- Group A: SER analysis with CFO variation for fixed pulse shaping filter and ABS altitude.

- Group B: SER analysis with ABS altitude variation for fixed CFO and pulse shaping filter.

- Group C: SER analysis with pulse shaping variation for fixed CFO and ABS altitude.

\section{A. Group A Results}

This section presents the GFDM performance analysis for different values of the CFO without estimation and compensation. Moreover, we chose "Fsech" as pulse shaping filter and set the ABS altitude to $500 \mathrm{~m}$, since these values resulted optimal in simulations of Group B and C. In GFDM, synchronization is a well-known problem which happens due to timing offset and CFO. Here we introduce CFO in our system model to calculate the SER variation. The CFO values chosen are realistic, simply to show the SER variation in different environments. A baseline result without CFO is also provided, to validate the results. We observe from Figs. 1 (a), (b) and (c) that SER increases with the increase in the value of CFO. For example, SER improvement of $1.92 \times 10^{-2}$ is observed at SNR of $30 \mathrm{~dB}$ with minimum and maximum considered CFO range in suburban environment. Similarly, $2.4 \times 10^{-2}$ and $9.4 \times 10^{-3}$ SER improvements were observed in urban and urban high rise environments, respectively with same values of CFO and SNR. This happens due to the introduction of ICI between adjacent sub-carrier in all environments.

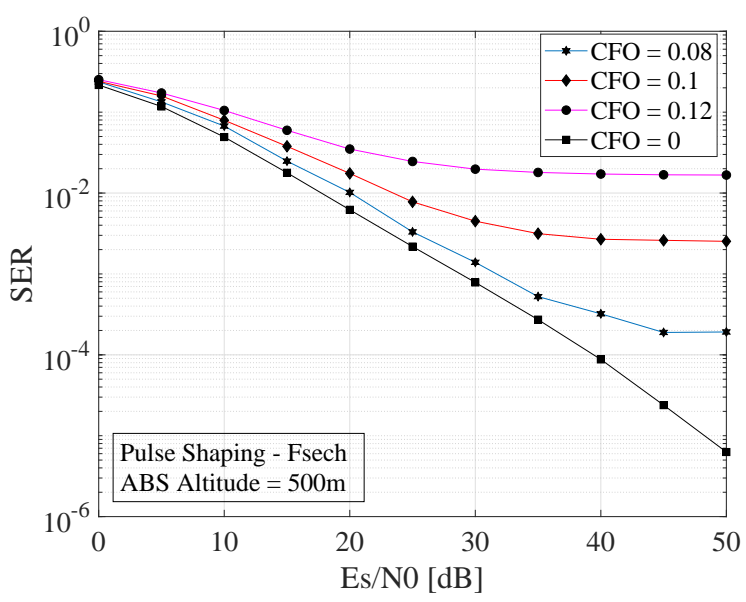

(a) Suburban Environment

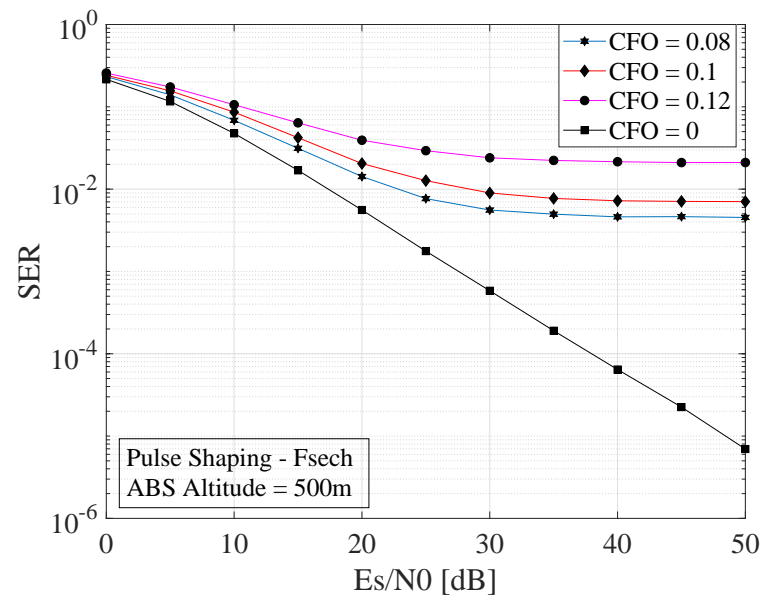

(b) Urban Environment

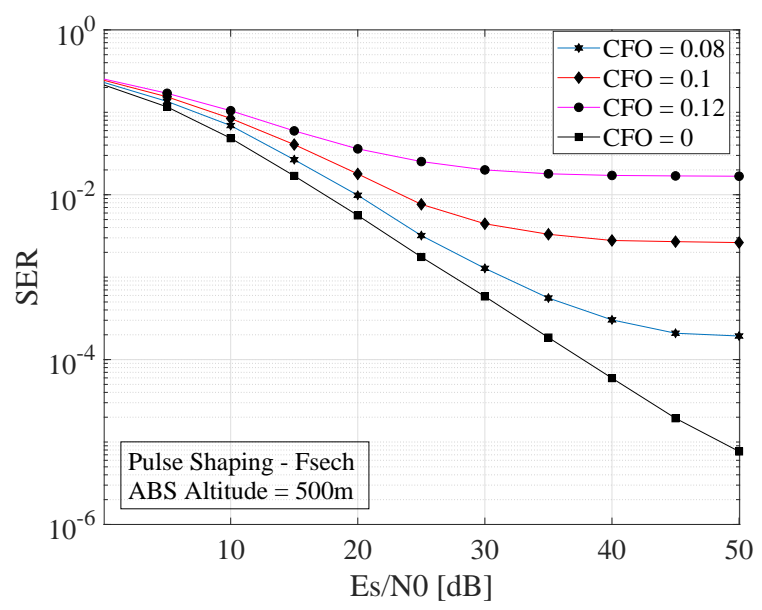

(c) Urban High Rise Environment

Fig. 1. Group A: SER vs. $E_{s} / N o$ performance analysis for fixed pulse shaping and ABS altitude with CFO variation (a) Suburban (b) Urban (c) Urban High Rise.

\section{B. Group B Results}

In this section, we present SER with "CFO $=0.1$ " and "Fsech" pulse shaping filter. We simulated ABS altitudes ranging from $100 \mathrm{~m}$ to $2000 \mathrm{~m}$. However, we show a certain number of values here. We observe that for lower altitudes of ABS, SER values tend to be higher. This is due to the presence 


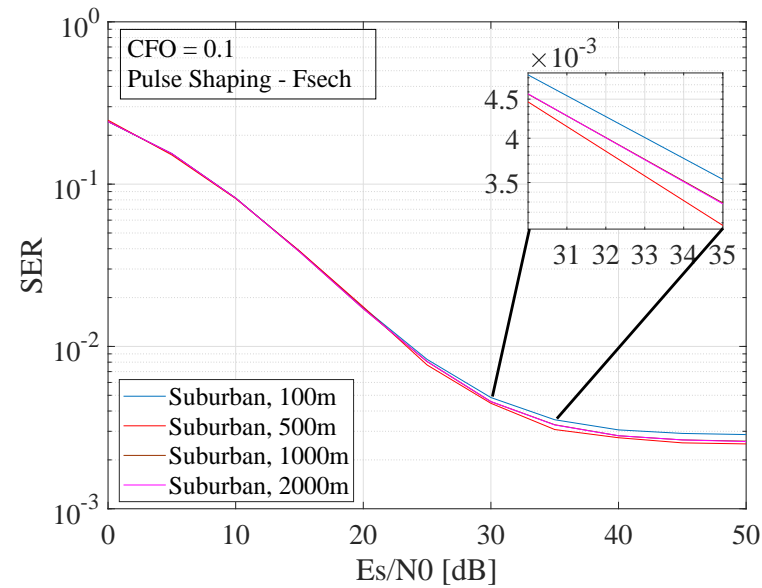

(a) Suburban Environment

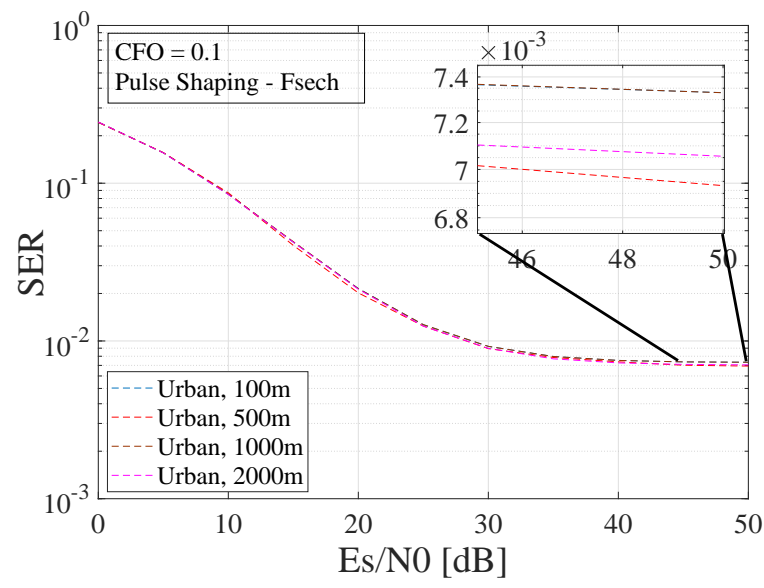

(b) Urban Environment

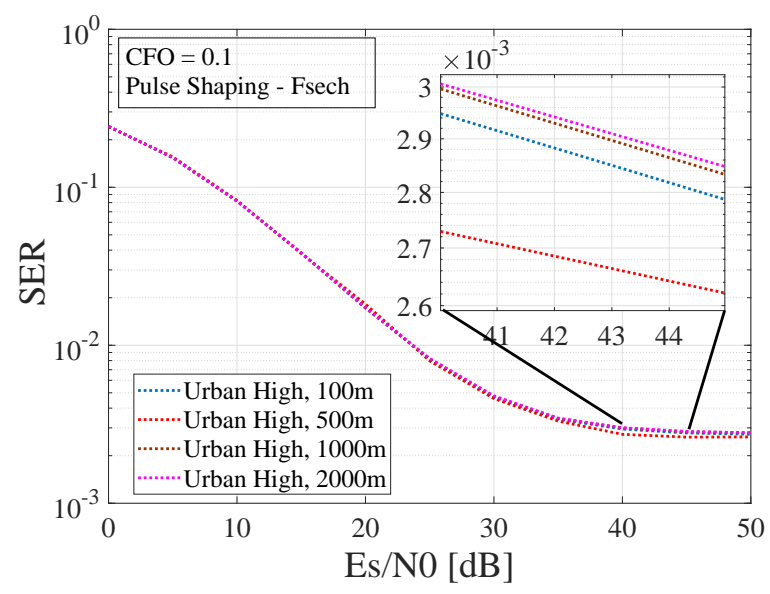

(c) Urban High Rise Environment

Fig. 2. Group B: SER vs. $E_{s} / N o$ performance analysis for fixed CFO and pulse shaping filter with ABS altitude variation.

of large multipath effects leading to large and small-scale fading effects and finally attenuation of the received signal strength of the ground user. Also at much higher altitudes, the SER was found to be higher, due to the large distance between ABS and ground user leading to large PL values, as shown in (3) and (4). Therefore, there exists a trade-off for an optimal altitude where minimum SER is obtained.

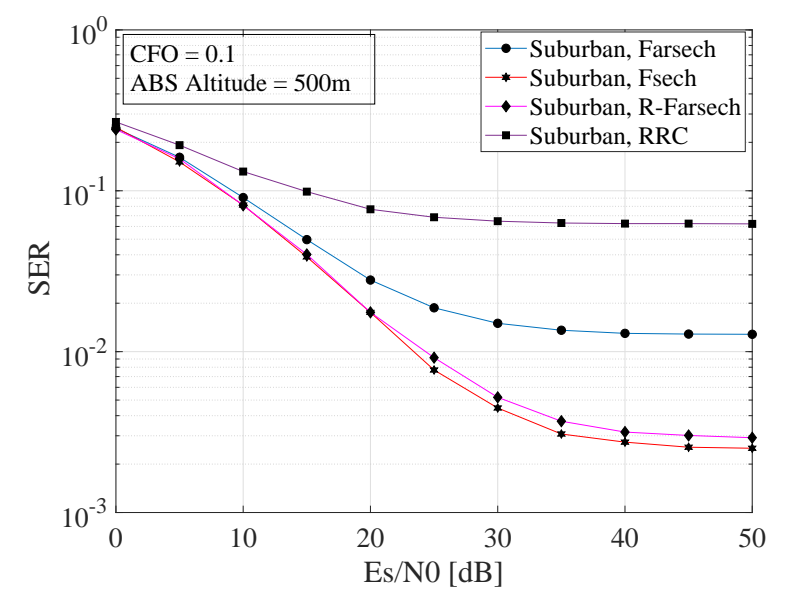

(a) Suburban Environment

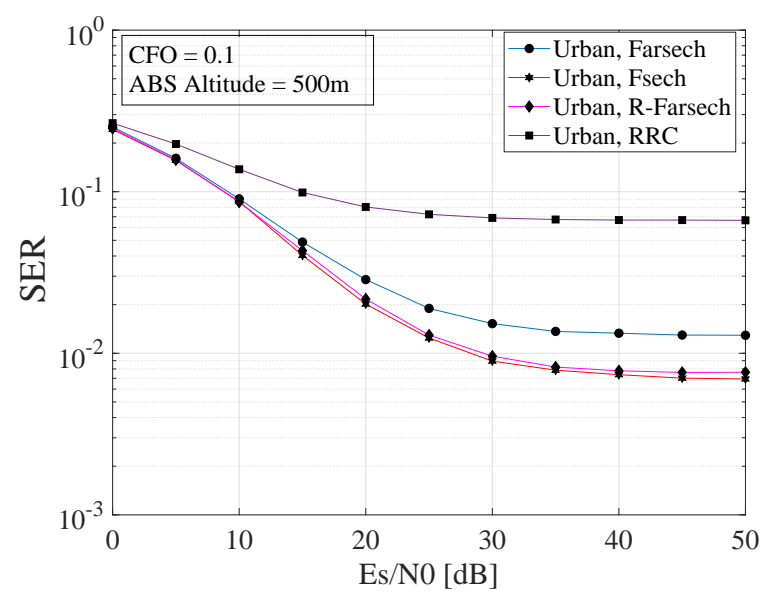

(b) Urban Environment

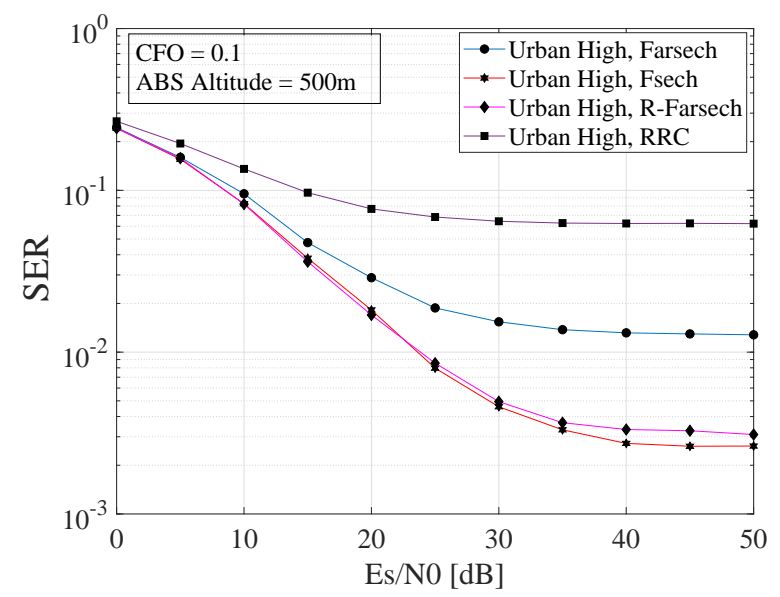

(c) Urban High Rise Environment

Fig. 3. Group C: SER vs. $E_{s} / N o$ performance analysis for fixed for pulse shaping variation.

From our simulations, we obtained minimum SER at 500m ABS altitude, as seen from Figs. 2 (a), (b) and (c) for Suburban, Urban and Urban High Rise scenario, respectively. We can also note that similar range of optimal altitude has been proposed in the literature for maximum cell coverage by the ABS [19], [24]. We also observe from the simulations, that SER variation is not high at different altitudes. For instance, 
SER improvements of $4 \times 10^{-4}, 3.5 \times 10^{-4}$ and $1.9 \times 10^{-4}$ between best and worst SER values were seen at $30 \mathrm{~dB}$ in suburban, urban and urban high rise environments. This is due to the frequency flat fading scenario observed in UAV communication.

\section{Group C Results}

In this section, we address the variation of SER with different BTN pulse shaping and RRC filters for " $\mathrm{CFO}=0.1$ " and "ABS altitude $=500 \mathrm{~m}$ ". We have implemented BTN pulse shaping for our work, since it proves to provide a lower OOB emission and better SER performance as compared to Nyquist ones, as seen from Figs. 3 (a), (b) and (c). This has been reported in [15], for Rayleigh fading channel, which is usually used for terrestrial communication. However, this is the first work implementing these filters in Ricean A2G channel model.

We observe from Figs. 3 (a), (b) and (c) for Suburban, Urban and Urban High Rise environments, respectively, that "Fsech" is an optimal BTN pulse shaping filter from the three considered ones with minimum SER. However, this is different from the case of Rayleigh fading, where minimum SER was obtained with "RRC" pulse shaping. The improvement in the use of BTN compared to root-raised cosine (RRC) is higher for A2G links compared to terrestrial links. This is due to predominance of LoS. As seen from Figs. 3 (a), (b) and (c), significant SER improvement in all three considered environments, around $6 \times 10^{-2}$, were observed between "Fsech" and "RRC" filters.

\section{CONCLUSiON}

In this article we address aerial base station (ABS) network serving ground mobile users in different environments such as suburban, urban and urban high rise. We performed SER calculations at different ABS altitudes ranging from $100-2000 \mathrm{~m}$ for GFDM system model with low latency communication in presence og CFO with "Better than Nyquist" pulse shaping filters. We addressed the variation of SER at different $\mathrm{CFO}$ values for optimal altitude of ABS. Our results provide realistic performance by using air-to-ground channel parameters obtained from commercial ray tracing software. The path loss values obtained were used to find the path gain or channel tap values of each multipath component.

\section{ACKNOWLEDGEMENT}

This project has received funding from the European Union Horizon 2020 research and innovation program under grant agreement No. 668995 and, in part from, Sri Lanka Technological Campus, Sri Lanka.

\section{REFERENCES}

[1] E. Dahlman, G. Mildh, S. Parkvall, J. Peisa, J. Sachs, Y. Selén, and J. Sköld, "5G wireless access: requirements and realization," in IEEE Communications Magazine, vol. 52, no. 12, pp. 42-47, December 2014.

[2] S. Naqvi, S. Hassan, H. Pervaiz, and Q. Ni, "Drone-Aided Communication as a Key Enabler for 5G and Resilient Public Safety Networks," in IEEE Communications Magazine, vol. 56, no. 1, pp. 36-42, Jan 2018.

[3] V. Sharma, M. Bennis, and R. Kumar, "UAV-Assisted Heterogeneous Networks for Capacity Enhancement," in IEEE Communications Letters, vol. 20, no. 6, pp. 1207-1210, June 2016.
[4] P. Yang, X. Cao, C. Yin, Z. Xiao, X. Xi, and D. Wu, "Proactive Drone-Cell Deployment: Overload Relief for a Cellular Network Under Flash Crowd Traffic," in IEEE Transactions on Intelligent Transportation Systems, vol. 18 , no. 10 , pp. 2877-2892, Oct 2017.

[5] N. Sharma, M. Magarini, D. N. K. Jayakody, V. Sharma, and J. Li, "OnDemand Ultra-Dense Cloud Drone Networks: Opportunities, Challenges and Benefits," in IEEE Communications Magazine, vol. 56, no. 8, pp. 85-91, August 2018.

[6] Z. Wu, H. Kumar, and A. Davari, "Performance evaluation of OFDM transmission in UAV wireless communication," in Proceedings of the Thirty-Seventh Southeastern Symposium on System Theory, SSST '05., March 2005, pp. 6-10.

[7] Q. Wu and R. Zhang, "Delay-constrained throughput maximization in UAV-enabled OFDM systems," in 23rd Asia-Pacific Conference on Communications (APCC), Dec 2017, pp. 1-6.

[8] C. Blümm, C. Heller, and R. Weigel, "SDR OFDM Waveform Design for a UGV/UAV Communication Scenario," Journal of Signal Processing Systems, vol. 69, no. 1, pp. 11-21, Oct 2012. [Online]. Available: https://doi.org/10.1007/s11265-011-0640-8

[9] J. Kakar and V. Marojevic, "Waveform and spectrum management for unmanned aerial systems beyond 2025," in IEEE 28th Annual International Symposium on Personal, Indoor, and Mobile Radio Communications (PIMRC), Oct 2017, pp. 1-5.

[10] H. Nishiyama, Y. Kawamoto, and D. Takaishi, "On OFDM-Based Resource Allocation in LTE Radio Management System for Unmanned Aerial Vehicles (UAVs)," in IEEE 86th Vehicular Technology Conference (VTC-Fall), Sept 2017, pp. 1-5.

[11] V. Vahidi and E. Saberinia, "OFDM for payload communications of UAS: channel estimation and ICI mitigation," in IET Communications, vol. 11, no. 15, pp. 2350-2356, 2017.

[12] M. Simsek, A. Aijaz, M. Dohler, J. Sachs, and G. Fettweis, "5G-Enabled Tactile Internet," in IEEE Journal on Selected Areas in Communications, vol. 34, no. 3, pp. 460-473, March 2016.

[13] I. Gaspar, L. Mendes, M. Matthé, N. Michailow, A. Festag, and G. Fettweis, "LTE-compatible 5G PHY based on generalized frequency division multiplexing," in 11th International Symposium on Wireless Communications Systems (ISWCS), Aug 2014, pp. 209-213.

[14] N. Sharma, M. Magarini, L. Dossi, L. Reggiani, and R. Nebuloni, "A study of channel model parameters for aerial base stations at $2.4 \mathrm{GHz}$ in different environments," in 15th IEEE Annual Consumer Communications Networking Conference (CCNC), Jan 2018, pp. 1-6.

[15] A. Kumar and M. Magarini, "Improved Nyquist pulse shaping filters for generalized frequency division multiplexing," in 8th IEEE Latin-American Conference on Communications (LATINCOM), Nov 2016, pp. 1-7.

[16] 3GPP TR 36.777 V15.0.0. (2017, Dec.) in Technical Specification Group Radio Access Network; Technical Specification Group Radio Access Network; Study on Enhanced LTE Support for Aerial Vehicles (Release $15)$.

[17] D. Zhang, A. Festag, and G. P. Fettweis, "Performance of Generalized Frequency Division Multiplexing Based Physical Layer in Vehicular Communications," in IEEE Transactions on Vehicular Technology, vol. 66, no. 11, pp. 9809-9824, Nov 2017.

[18] N. Michailow, M. Matthé, I. S. Gaspar, A. N. Caldevilla, L. L. Mendes, A. Festag, and G. Fettweis, "Generalized Frequency Division Multiplexing for 5th Generation Cellular Networks," in IEEE Transactions on Communications, vol. 62, no. 9, pp. 3045-3061, Sept 2014.

[19] A. Al-Hourani, S. Kandeepan, and S. Lardner, "Optimal LAP Altitude for Maximum Coverage," in IEEE Wireless Communications Letters, vol. 3, no. 6, pp. 569-572, Dec 2014.

[20] W. Khawaja, I. Guvenc, D. Matolak, U.-C. Fiebig, and N. Schneckenberger, "A Survey of Air-to-Ground Propagation Channel Modeling for Unmanned Aerial Vehicles," ArXiv e-prints, Jan. 2018.

[21] A. Kumar, M. Magarini, and S. Bregni, "Impact of "Better than Nyquist" pulse shaping in GFDM PHY with LTE-compatible frame structure," in IEEE 9th Latin-American Conference on Communications (LATINCOM), Nov 2017, pp. 1-6.

[22] "Propagation data and prediction methods for the design of terrestrial broadband millimetric radio access systems," in Geneva, Switzerland, Rec. P.1410-2, 2003, P Series, Radiowave Propagation.

[23] t. P. Mededovid, "Wireless insite software verification via analysis and comparison of simulation and measurement results," in Proceedings of the 35th International Convention MIPRO, May 2012, pp. 776-781.

[24] D. G. Cileo, N. Sharma, and M. Magarini, "Coverage, capacity and interference analysis for an aerial base station in different environments," in International Symposium on Wireless Communication Systems (ISWCS), Aug 2017, pp. 281-286. 